\title{
Robotic ureter reconstruction using the native ureter to treat long-segment ureteral stricture of the transplant kidney: the first Korean experience
}

\author{
Jinu Kim ${ }^{1}$, Seok Jeong Yang ${ }^{2}$, Deok Gie Kim ${ }^{3}$, Woong Kyu Han${ }^{1}$, Joon Chae $\mathrm{Na}^{4}$ \\ ${ }^{1}$ Department of Urology, Severance Hospital, Seoul, Korea \\ ${ }^{2}$ Department of Surgery, Yongin Severance Hospital, Yongin, Korea \\ ${ }^{3}$ Department of Surgery, Wonju Severance Christian Hospital, Wonju, Korea \\ ${ }^{4}$ Department of Urology, Yongin Severance Hospital, Yongin, Korea
}

Background: The complication of the ureter after kidney transplantation (KT) has been reported to occur in $2.6 \%$ to $15 \%$ and ureteral stricture is one of the common complications. We report two cases of robotic ureteral reconstruction surgery to correct the long-segment ureteral stricture of the transplanted kidney.

Methods: After docking the Da Vinci Xi the transplanted ureter was dissected and the stricture point was identified. Nephrectomy of the right kidney was performed and the native ureter was dissected. An end-to-side anastomosis of the native ureter to transplant ureter was done. Indocyanine green (ICG) was used to aid in identifying the transplant ureter and also to confirm the vascularity of the dissected ureter.

Results: Case 1: A 55-year-old female developed ureter stricture 5 months after deceased donor kidney transplant (DDKT) and was referred to our hospital after recurrent episodes of febrile urinary tract infection (UTI), and her serum creatinine was 3.01 $\mathrm{mg} / \mathrm{dL} 16$ months post $\mathrm{KT}$. After robotic reconstruction, she had one episode of febrile UTI 3 months after surgery and had no more episodes afterward. The serum creatinine after 1 year was $2.42 \mathrm{mg} / \mathrm{dL}$. Case 2: A 56-year-old female developed ureter stricture 1 month after DDKT. The patient was referred to our hospital 3 months after KT for ureter reconstruction. Her serum creatinine was $1.20 \mathrm{mg} / \mathrm{dL}$ before surgery and was $1.31 \mathrm{mg} / \mathrm{dL}$ at 9 months follow-up.

Conclusions: We report two cases of robotic uretero-ureterostomy using the native ureter for the reconstruction of a long-segment ureteral stricture of the transplanted kidney. This was the first attempt in Korea to use the robot to manage urologic complications after KT. The procedure is safe, feasible and using the robot provides advantages by utilizing ICG to identify the transplant ureter and confirm the viability of the graft ureter.

Corresponding author: Joon Chae $\mathrm{Na}$

E-mail:URONA@yuhs.ac

(c) The Korean Society for Transplantation

This is an Open Access article distributed under the terms of the Creative Commons Attribution Non-Commercial License (http://creativecommons.org/licenses/by-nc/4.0/) which permits unrestricted non-commercial use, distribution, and reproduction in any medium, provided the original work is properly cited. 